\title{
LOS ESPACIOS DE PRODUCCIÓN DE CAFÉ SUSTENTABLE EN MÉXICO EN LOS INICIOS DEL SIGLO XXI
}

Pablo Pérez Akaki

ppablo@apolo.acatlan.unam.mx

FACUltad DE Estudios SuPERIORES, ACATLÁN

UNIVERSIDAD NACIONAL AuTÓNOMA DE MÉXICO

\section{RESUMEN}

Este documento analiza los diferentes sistemas de producción cafetaleros que han cobrado importancia en el ámbito internacional a los que usualmente se les llama alternativos y sustentables, sin cuestionarse suficiente sobre el concepto mismo de sustentabilidad. Al revisar la evolución de estos sistemas a escala mundial, es claro que en ellos se presentan numerosas contradicciones que generan varias interrogantes sobre las bondades que anuncian. Además se analiza cómo estos han cobrado importancia en México y se realiza un esfuerzo de cuantificación de su importancia. Se presenta finalmente un caso que muestra que los sistemas alternativos son importantes, pero limitados para conseguir al menos la suficiencia económica que demandan los productores.

Palabras clave: Sistemas alternativos, manejo sustentable, cooperativas, cafeticultura. 


\begin{abstract}
This document analyzes the different coffee production systems that have gained importance in the international sphere which are commonly referred to as alternative and sustainable, without sufficient questioning of the concept itself of sustainability. Upon reviewing the evolution of these systems at the world scale, it is clear that numerous contradictions are presented in them that generate various questions on the virtues they extol. The document also analyzes how they have acquired importance in Mexico and an effort is made to quantify the same. Finally, a case is presented that demonstrates that the alternative systems are significant but limited in their procurement of at least the economic sufficiency demanded by producers.
\end{abstract}

Key words: Alternative systems, sustainable management, cooperatives, coffee production. 


\section{INTRODUCCIÓN}

En los últimos años se han registrado cambios en lo que se llama el modo de acumulación económica hacia un sistema posfordista caracterizado por formas de producción flexibles (Boyer 2002: 233), de nicho de mercado, de productos con alto valor agregado y, en el caso agroalimentario, caracterizado por productos frescos y sanos, en un entorno altamente competitivo, con la producción distribuida geográficamente en todo el mundo y guiada por el sector privado (Busch y Bain 2004: 322-325). Esto significa que tanto la agricultura como la producción de alimentos se integran en un conjunto de procesos trasnacionales e intersectoriales (Murdoch, Marsden y Banks 2000: 111).

En este nuevo contexto, el valor de las exportaciones desde los países tropicales de los llamados productos tradicionales disminuyó — café, algodón, cacao, caña de azúcar- y otros productos cobraron importancia, representando las proporciones más altas del valor total del comercio internacional de alimentos: frutas, hortalizas, lácteos, carnes, mariscos, entre los principales (Watts y Goodman 1997).

Estas nuevas demandas han impulsado trasformaciones en los sistemas de producción pues, además de que las empresas enfrentan mayor desregulación y apertura en los mercados, son parte de importantes cambios institucionales caracterizados por la integración vertical y el fortalecimiento de la propiedad privada, en un entorno altamente competitivo empujado por los cambios tecnológicos, entre ellos la biotecnología, los sistemas de comunicación y trasporte, los procesos industriales y demás (Reardon y Barret 2000: 197). De esta manera, las empresas de este sector fueron testigos de concentración y oligopolización en los procesos industriales, en el comercio, en la distribución y en el 
financiamiento (Renard 1999: 150-160). Particularmente en el caso del café, el cambio en el modo de acumulación significó la emergencia de nuevos sistemas de producción, comercialización y consumo, entre los que destacan el café de especialidad, los orgánicos, el comercio justo, los ecológicos, los amigables con las aves migratorias y los responsables, entre los más comunes. El café de especialidad representa una alternativa centrada en la mejora de la calidad en todas las fases del proceso como un criterio de diferenciación, desde que el grano se cultiva hasta que se consume, a lo que se agrega el «refinamiento, calidad y buen ambiente» (Pendergrast 2002: 357, Rindova y Fombrun 2001: 237). El resto de los sistemas tienen un enfoque más amplio pues incluyen dimensiones ambientales, sociales y económicas.

Una de las posturas utilizadas para el análisis de estas trasformaciones es la teoría de las convenciones. Una convención es un patrón de comportamiento que representa una costumbre, es esperado y se autorefuerza con las interacciones (Young 1993: 57). Este término es utilizado para denotar los «paradigmas sociales», que se generan a partir de la interacción cotidiana entre actores sociales, y que son utilizados para minimizar riesgos y están presentes en todas las interacciones humanas (Choi 1999: 255). Según la economía, una convención representa un acuerdo al que llegan los participantes en un proceso que se repite muchas veces, en donde todos están de acuerdo y todos esperan que el resto de los participantes estén de acuerdo (Lewis 1969); es decir, un equilibrio en la interacción entre agentes que tienen un comportamiento maximizador de utilidad.

Algunos sostienen incluso que este equilibrio es eficiente en el sentido de Pareto, aun cuando existan comportamientos con racionalidad limitada, tales como un comportamiento evolutivo de los agentes o una corta visión en la elección de las estrategias 
para la interacción, pero debe cumplirse la condición de que los actores participantes tengan cierto nivel de coordinación o un interés en común (Young 1993, Oechssler 1999).

Un régimen estable de convenciones tiene importancia para el desarrollo humano y social porque ofrece oportunidades para el emprendimiento de nuevos proyectos económicos (Choi 1999: 258). Además las convenciones pueden funcionar en diferentes sentidos, promoviendo o eliminando la competencia, estabilizando los mercados alrededor de ciertas ideas establecidas, tecnologías, actividades o prácticas morales (Biggart y Beamish 2003: 453). Para ello se requiere un ambiente que propicie la innovación (Choi 1999: 258), lo que implica la existencia de una serie de instituciones y organizaciones que ofrezcan el marco necesario para este propósito.

En este sentido, las instituciones se definen como conjuntos de convenciones que han emergido al resolver problemas cotidianos y que son adoptadas como «lo normal» (Biggart y Beamish 2003: 458). Particularmente en el caso del café, la teoría de las convenciones ha sido utilizada por Ponte (2002), Raynolds (2002a, 2002b), Daviron y Ponte (2005), Giovannucci y Ponte (2005), Kolk (2005), Muradian y Pelupessy (2005) entre otros, para estudiar las características de las cadenas comerciales de café, tanto tradicionales como las emergentes.

Así, el objetivo de este trabajo es ofrecer un análisis de los sistemas alternativos de producción, comercialización y consumo de café, y su importancia en el espacio productivo mexicano. Para ello, en la segunda parte del trabajo se discute sobre los diferentes sistemas alternativos, denominados por algunos sustentables. La tercera parte particulariza hacia la importancia que estos tienen en México, y la última presenta el análisis de un caso mexicano. 
Merece la pena aclarar que el concepto de espacio productivo de café mexicano está vinculado de manera directa con los elementos característicos de los lugares donde esta actividad sucede, tanto en sus aspectos físicos como económicos y sociales.

\section{LOS SISTEMAS ALTERNATIVOS DE PRODUCCIÓN, COMERCIALIZACIÓN Y CONSUMO DE CAFÉ}

Al hablar sobre los sistemas alternativos de producción, comercialización y consumo de café, se identifican en este grupo el café orgánico, el comercio justo, el ecológico, el amistoso con las aves migratorias, el responsable y el Common Code, ${ }^{1}$ alternativas que emergieron en los últimos años del periodo de cuotas o en los primeros del periodo de liberalización que le siguió. En todos ellos, los actores participan voluntariamente y existen terceras instancias que se encargan de la certificación de los procesos (Muradian y Pelupessy 2005: 2032-2036). A continuación se discute sobre cada uno de ellos, dimensionando su importancia mundial y destacando sus diferencias.

\section{CAFÉ ORGÁNICO \\ El café orgánico se define como}

un sistema holístico de gestión de la producción que fomenta y mejora la salud de los agroecosistemas, y en particular la biodiversidad, los ciclos biológicos y la actividad biológica del suelo, a través de prácticas que evitan el uso de productos de síntesis química, así como de organismos genéticamente modificados, aguas negras, edulcorantes y conservadores sintéticos en productos transformados (Gómez Cruz et al. 2005: 11). 
De acuerdo con la International Federation of Organic Agriculture Movements, IFOAM, organismo con sede en Alemania, incluye «todos los sistemas agrícolas que promueven la producción de alimentos y fibras con una viabilidad ambiental, social y económica», y está basado en cuatro principios: en la salud del suelo, las plantas, las personas y el planeta como un único ente que es indivisible; en la promoción y conservación de los ecosistemas y ciclos ecológicos; en la equidad, que implica que se basa en relaciones de igualdad para los humanos y el medio ambiente; y en la precaución, pues no compromete el bienestar de las sociedades presentes ni futuras ni el del medio ambiente (IFOAM 2005).

Operativamente, el sistema orgánico es un proceso de certificación que los productores de café obtienen a partir del cumplimiento de ciertos requisitos para el control de plagas mediante técnicas naturales de equilibrio biológico, manejo adecuado del cultivo y de uso de medidas fitosanitarias. También establece requisitos para el reciclaje de material orgánico y su uso como abonos, para la promoción y aprovechamiento de la biodiversidad, cuidado de la sombra que afecte a los cultivos, la promoción de cubiertas vegetales y la promoción de la rotación de cultivos. ${ }^{2}$

Este sistema productivo compromete a los participantes a respetar las leyes laborales internacionalmente aceptadas, entre ellas la no discriminación, prohibición del trabajo infantil, libertad de asociación y respeto a los salarios mínimos, entre otras. No establece una restricción por el tamaño de la unidad productiva ni restringe el comportamiento comercial de las empresas participantes.

La importancia del café orgánico en el ámbito mundial aumentó aceleradamente desde la década de los ochenta, década en la que aparecieron algunas de las agencias certificadoras más relevantes para el contexto mexicano, como Naturland y OCIA. ${ }^{3}$ Ya para 
1992 se registró un consumo mundial de 24,000 sacos orgánicos (ICO 1997), mientras que en 2005 las exportaciones reportadas por la International Coffee Organization, ICO, fueron de 797,616 sacos orgánicos (ICO 2006). Estas cifras muestran un crecimiento anual promedio de $31 \%$ en dicho periodo.

\section{CAFÉ DE COMERCIO JUSTO}

El comercio justo representa un movimiento surgido desde principios del siglo XX bajo la demanda de equidad en el comercio internacional. Este modelo propuso que para conseguir la equidad se debía mantener un sistema de cuotas, lográndose así consolidar a International Coffee Organization; tras la terminación de dicho sistema, el movimiento de comercio justo derivó en el sistema de sellos (Fridell 2006: 45), lo que aconteció durante la década de los ochenta. Esta nueva estrategia significó que la red de comercio justo lograra un mejor posicionamiento, lo que se manifestó en las altas tasas de crecimiento del valor de las exportaciones, superando $20 \%$ en los primeros años del siglo XXI, ${ }^{4}$ y destinando más de 60\% al mercado europeo. Este representó ventas superiores a casi seiscientos millones de euros en 2004, y los productos de mayor valor fueron café y plátano, por orden de importancia (Krier 2005: 7).

El comercio justo se coordina internacionalmente por medio de Fairtrade Labelling Organizations, FLO, International, institución con sede en Alemania fundada en los noventa, que funciona como una federación para coordinar los esfuerzos de diferentes instituciones nacionales, entre las que se encuentran Transfair, EUA; Max Havelaar, Holanda; GEPA, Alemania; Oxfam, Francia y Reino Unido; Comercio Justo México, entre otras. 
Según FLO, este sistema se define como una sociedad de comercio basada en el diálogo, la trasparencia y el respeto, que busca mayor equidad en el comercio internacional. Sus objetivos son conseguir la mejoría en las condiciones de vida de los más pobres y marginados, el fortalecimiento del papel de los productores y empleados, y difundir los esfuerzos de justicia a una escala global (Krier 2005: 21). Este sistema se identifica como un sistema de etiquetas —labels — para la identificación del producto.

La propuesta de comercio justo implica responsabilidades para todos los actores en la cadena de comercialización, lo cual tiene como objetivo garantizar relaciones comerciales de largo plazo y una mayor equidad para todos los participantes, mejorando su acceso al mercado y fortaleciendo su organización colectiva (Giovannucci y Koekoek 2003: 38).

\section{CAFÉ ECOLÓGICO}

La importancia como sistema de producción del café ecológico, en algunos casos llamado café de sombra, se acrecentó como una respuesta ante los daños ecológicos provocados por el ser humano. Estos daños se han acumulado a lo largo del tiempo y se manifiestan en la reducción de la flora y fauna, en la contaminación de los suelos y la fragmentación de los ecosistemas (Rice y Ward 1996: 6).

El objetivo concreto del café ecológico es la protección de los predios cafetaleros bajo sombra porque son refugio para la biodiversidad, particularmente para algunas aves migratorias, como por ejemplo orioles de Baltimore, Icterus galbula, y chipes peregrinos, Vermivora peregina (Perfecto, Rice, Greenberg y Van der Voort 1996). Esta mayor diversidad se encontró en los predios cafetaleros con una producción tradicional, donde se 
combinó con otros productos — multicultivo— y se mantuvo una estructura de árboles de distinto tamaño. Dentro de esta categoría existen dos iniciativas: la de Smithsonian Migratory Bird Center (SMCB 2007), propuesta que surgió durante los noventa, y la de Rainforest Alliance, que inició en 1987, la cual tiene como objetivo la protección del ambiente mediante la práctica de una agricultura sustentable, incluyendo los agentes en la cadena de comercialización (Rainforest Alliance 2006a).

Para SMCB, un café sustentable se produce en «fincas con una importante biodiversidad biológica y un bajo uso de insumos, pues conserva recursos, protege el ambiente, produce con eficiencia, compite comercialmente y aumenta la calidad de vida de los productores y la sociedad en conjunto» (Greenberg 2001: 1). Esto implica que el grano debe ser producido utilizando técnicas que protegen los suelos, la diversidad biológica, los mantos acuíferos, promoviendo la captura de carbono, la reforestación, y con uso mínimo de productos químicos. Los productores deben tener acceso a la información de mercado, a créditos justos, con producción diversificada, donde se cumpla con estándares internacionales de calidad observados por terceras instancias, que aseguren el respeto de las prácticas culturales locales (Greenberg 2001, SMBC 2002). Sin embargo, en la práctica, las normas utilizadas para la certificación solo establecen criterios para la diversidad biológica en densidad y composición de árboles de sombra para los predios cafetaleros, manteniendo los criterios generales para la certificación de producción orgánica. No establece criterios sociales que deben cumplirse por los participantes, sino que confía en el mercado para la asignación de esta tarea.

Por el otro lado, Rainforest Alliance ha propuesto una certificación con criterios muy específicos junto con un conjunto de organizaciones civiles que conformaron la Red 
para la Agricultura Sustentable, SAN, ${ }^{5}$ donde se establecen criterios para la sustentabilidad que están basados en una serie de principios, algunos obligatorios, sobre buenas prácticas productivas y administrativas, conservación de la biodiversidad, los suelos y los mantos acuíferos, impulso a los sistemas de producción integrados, buenas prácticas de contratación para los trabajadores: justas, seguras y saludables, entre otras. Sin embargo, el énfasis está puesto en los aspectos biológicos y de cumplimiento de los estándares laborales, descuidando el resto: no hay beneficios directos como la prima social, las relaciones de largo plazo y los financiamientos. Este sistema además permite el uso de ciertos agroquímicos, lo que es una diferencia muy importante respecto al orgánico y al café amistoso con las aves migratorias. Ambos son sistemas de etiquetas —labels — para la identificación del producto en los mercados de consumo.

El impacto del sistema de SMBC es de 36 fincas, en 7 países, donde se incluyen 2,372 productores a lo largo de una superficie de 7,610 hectáreas. La producción alcanzada en este espacio es de aproximadamente 3,637 toneladas de café (SMBC 2007). En el caso de Rainforest Alliance, la superficie certificada de café hasta enero de 2007 fue de 64,000 hectáreas (Rainforest Alliance 2007b), vinculando a 264 organizaciones que representaron una producción ligeramente superior a 121,000 toneladas (Rainforest Alliance 2007c).

\section{CAFÉ RESPONSABLE}

La certificación de café bajo la figura Utz Kapeh, «buen café» en lengua maya, se trata de un programa basado en la producción y comercialización responsable del grano, orientado a identificar el origen del café, la ruta de comercio hasta el consumidor y las condiciones en 
las que fue producido. El modelo surgió como una iniciativa entre productores de Guatemala y comercializadores en Holanda, y en este último figura la sede del programa. Las normas para los productores se organizan en cuatro grupos: criterios productivos sanos, entre los cuales se especifica que deben existir procesos de monitoreo, registro de aplicación de fertilizantes, entrenamiento de los empleados, reglas de acción en caso de accidentes, normas sanitarias para el proceso y registro de todas las actividades a desarrollar. En segundo lugar, criterios sociales y ambientales, que consisten, por un lado, en el respeto a las leyes laborales internacionales, permitir el acceso a los sistemas de salud, educación para los menores, vivienda digna; y por el otro, la reducción de la erosión del suelo, minimización del uso de agroquímicos y del uso de energía, uso eficiente del agua, evitar la deforestación y proteger a especies en peligro de extinción. En tercer lugar, el rastreo, que permite la asignación de responsabilidades para cada uno de los participantes en la cadena de producción y comercialización. El cuarto, probablemente el criterio más difícil de cumplir para los pequeños productores, el manejo administrativo, que significa tener registros de todas las operaciones realizadas, tanto comerciales como productivas (Utz Kapeh 2006a, Gamboa 2007).

Para los productores, la ventaja de esta certificación es que el café podría venderse por arriba del precio que se percibe en el mercado tradicional, aunque esto no está garantizado. Para los compradores, entre ellos los consumidores, la información significa seguridad y certidumbre en las características del grano, así como un estricto cuidado durante el traslado a los espacios de consumo.

Desde su inicio en 1997, la iniciativa ha tenido un crecimiento acelerado: al cierre de 2005, las organizaciones productoras certificadas fueron 135 en 16 países, lo que 
representó un total de casi 29,000 toneladas de café. Frente a esta oferta existió una demanda compuesta por 50 compradores registrados que adquirieron 480,000 sacos en dicho periodo (Utz Kapeh 2006b: 12). Las tasas de crecimiento tanto en el número de productores certificados como en los volúmenes de comercialización bajo este sistema superan $30 \%$ anual, y los pequeños productores tienen una participación menor a $40 \%$ (Gamboa 2007).

\section{CÓDIGO COMÚN PARA LA COMUNIDAD CAFETALERA}

Mejor conocido como 4C, el Código Común para la Comunidad Cafetalera, Common Code for the Coffee Community Asociation, es un código de conducta desarrollado por una organización en la que coinciden todos los participantes del sector cafetalero, con el objetivo de «aumentar continuamente la sustentabilidad en la producción, trasformación y comercialización de todo el café verde»(Common Code 2004: 1). El arranque de esta iniciativa se registró en 2003, y para 2006 el 4C se convirtió en un organismo privado con sede en Alemania.

De manera similar al sistema anterior, $4 \mathrm{C}$ se orienta a los mercados masivos de café, por ello desarrolló códigos de conducta para que todos los agentes de la cadena puedan alcanzar los criterios para la sustentabilidad, además de que se trata de un proceso dinámico que implica una mejora continua. Sus objetivos son mejorar la eficiencia, reducir costos, mejorar la calidad del grano, aumentar la productividad de todos los participantes de la cadena, facilitar el acceso al mercado de productores, aumentar el acceso a los créditos, trasparentar el mercado y aumentar la rentabilidad. Su estructura se configuró de manera tripartita entre tres grupos: productores, comercializadores e industriales, y sociedad civil, 
de tal manera que fuera capaz de recoger los intereses de todos los participantes en el sector.

Entre las normas establecidas, existen diez prácticas que son totalmente inaceptables: el trabajo infantil; el trabajo forzoso; servidumbre por deudas; tráfico de personas; prohibición del derecho a la organización sindical y negociación colectiva; desalojo forzoso; falta de provisión de agua potable y alojamiento en caso necesario; tala de bosques; uso de pesticidas prohibidos, y transacciones inmorales de negocios (Common Code 2004: 13-14).

\section{VENTAJAS Y DESVENTAJAS DE LOS SISTEMAS CAFETALEROS}

\section{ALTERNATIVOS}

Los análisis de numerosos estudios sostienen que los sistemas alternativos fortalecen las organizaciones de productores — pues por estos medios logran alcanzar mayores recursos en la comercialización de sus productos-, mejoran el ambiente, aumentan la inversión tecnológica y la innovación en las regiones productivas, se consigue disminuir la migración y aumentar la cohesión social, entre otros aspectos. Estos resultados pueden ser muy alentadores para que los no participantes se involucren en algunos de ellos; pero hay numerosas críticas, a continuación se presentan.

Con el café orgánico los productores podrían beneficiarse por: evitar el uso de tecnologías de producción de alto costo; evitar incurrir en adeudos con organismos financieros; la apertura a los mercados internacionales y nacionales de mayor valor agregado; el aumento del control sobre el proceso productivo; y el aumento en la confianza de los propios productores y la organización de redes sociales (Kilcher 2005: 49-50). 
También sería posible construir un modelo de cooperación entre los productores basado en la confianza y el conocimiento tácito, trasmitido por interacción directa entre los actores (Morgan y Murdoch 2000: 166-167), lo que permitiría el aumento en el capital social de las regiones productoras, revaloraría el conocimiento, los ecosistemas y las identidades locales, que trasformaría a los actores locales en figuras clave en el proceso productivo (Morgan y Murdoch 2000: 171).

Sin embargo hay importantes desventajas, como la imposibilidad de aislarse completamente del mercado, con lo cual los precios seguirán determinados tanto por las fuerzas económicas como por la especulación. También es peligroso el crecimiento tan importante en la demanda que ha llevado al olvido los principios holísticos que originaron el sistema, al grado de trasformarse en un factor de diferenciación en el mercado para conseguir un sobreprecio. La intervención del sector público es otro factor adicional que ha influido negativamente (González y Nigh 2005: 454-458).

Con el café bajo sombra se reduce la incertidumbre, pues su producción está diversificada en bienes agrícolas para autoconsumo y para los mercados locales (Rice y Ward 1996: 9). Los productores alcanzan un importante rango de sofisticación en cuanto a la administración de los suelos, pues hacen eficientes los espacios agrícolas al elegir los productos más adecuados a la vocación del suelo, utilizan materiales orgánicos y técnicas productivas acordes con la inclinación de la superficie (Rice y Ward 1996: 20). Como una importante desventaja puede decirse que los verdaderos motivadores del café de sombra no son los productores, sino la flora y fauna de los espacios cafetaleros, lo cual puede causar conflictos entre productores y los impulsores del sistema (González y Nigh 2005: 455-456). 
En el caso del café ecológico, la ventaja más importante es el mejoramiento de las prácticas productivas en materia agrícola, lo cual redundará en la calidad de la producción. Su principal desventaja radica, al igual que en el café de sombra, que el énfasis está en la dimensión ambiental de la sustentabilidad, dejando descuidadas las dimensiones económica y social.

En el caso del café responsable Utz Kapeh, su principal ventaja es que está orientado al mejoramiento productivo de la cadena principal de café, es decir, no tiene una orientación de nicho de mercado sino de los grandes volúmenes de comercio del grano. Esta misma condición representa la mayor desventaja para los pequeños productores, pues el sistema de comercialización descansa en los actores tradicionales y la posibilidad de escalamiento vertical está bien definida, y por tanto limitada.

Igual situación enfrenta el sistema de comercialización del Código Común, cuya orientación se da igualmente hacia el mercado tradicional y por ello confía en los actores tradicionales de comercialización y trasformación del grano. En una declaración a finales de 2006, directivos de Naturland y Gesellschaft zur Förderung der Partnerschaft mit der Dritten Welt mbH, GEPA, sostuvieron que 4C representa exigencias para los productores sin ninguna recompensa, lo cual llevaría a serios cuestionamientos sobre la manera de concebir el sistema productivo y la mejoría de las condiciones de los productores.

Todos los sistemas tienen como objetivo alcanzar la sustentabilidad, algunos apuntan a nichos específicos, mientras que otros intentan ser generales. Algunos ponen un énfasis en aspectos ambientales, otros en sociales y varios más en administrativos. Por ello en este documento se realiza una clasificación de los sistemas alternativos en seis grupos, dependiendo de los criterios de evaluación: productivos, que significa poner énfasis en la 
calidad de los procesos de producción, principalmente agrícola; administrativos, que comprenden la documentación y registro de las actividades realizadas en la unidad productiva; comerciales, que implican normas para las operaciones de compra-venta entre los agentes de la cadena; laborales, donde básicamente se consideran aspectos de respeto a los derechos laborales expresados por la Organización Internacional del Trabajo que giran en torno a la libertad de asociación, el respeto a la jornada laboral, la dignidad del trabajo, la no discriminación, entre otros; ambientales, donde el énfasis está puesto en el ambiente y los efectos de la actividad productiva sobre éste; y por último los sociales, que contemplan aspectos para el desarrollo de la sociedad en su conjunto. Estos se identificaron en el Cuadro 1 con tres categorías según su escala de exigencia: máxima, mediana o nula. Solo habrá un máximo en cada caso, que corresponde al de rangos más altos de exigencia; mediano significa que se tienen algunos criterios a ese respecto, y nulo que no hay mención alguna sobre este tema en los criterios de certificación.

Cuadro 1. Los sistemas alternativos y sus ejes fundamentales

\begin{tabular}{ccccccc}
$\begin{array}{c}\text { Dimensión de la } \\
\text { norma }\end{array}$ & Orgánico & $\begin{array}{c}\text { Comercio } \\
\text { justo }\end{array}$ & $\begin{array}{c}\text { Smithsonian } \\
\text { Migratory Bird } \\
\text { Center }\end{array}$ & $\begin{array}{c}\text { Rainforest } \\
\text { Alliance }\end{array}$ & $\begin{array}{c}\text { Utz } \\
\text { Kapeh }\end{array}$ & $\begin{array}{c}\text { Common } \\
\text { Code }\end{array}$ \\
\hline $\begin{array}{c}\text { Productivas } \\
\text { Administrativas }\end{array}$ & $\checkmark$ & $\checkmark$ & $\checkmark$ & $\checkmark$ & $\checkmark \checkmark$ & $\checkmark$ \\
Comerciales & $\mathrm{X}$ & $\checkmark$ & X & X & X & $\checkmark$ \\
Laborales & $\checkmark$ & $\checkmark \checkmark$ & X & $\checkmark$ & $\checkmark$ & $\checkmark$ \\
Ambientales & $\checkmark$ & $\checkmark$ & $\checkmark \checkmark$ & $\checkmark \checkmark$ & $\checkmark$ & $\checkmark$ \\
Sociales & X & $\checkmark \checkmark$ & X & $\checkmark$ & X & X \\
\hline
\end{tabular}

Nota: $\checkmark \checkmark$ - máximos requisitos, $\checkmark$ - algunos requisitos, $X$ - ningún requisito.

Fuentes: Naturland, 2005; FLO, 2007a; Smithsonian, 2002; Rainforest, 2005; Utz Kapeh, 2006a; Common Code, 2004.

Entre los sistemas, el de comercio justo se originó con el objetivo de crear redes alternativas de pequeños agentes que contrarrestaran las redes tradicionales fortalecidas por 
décadas, mientras que el resto apunta a incorporar a grandes empresas. Sin embargo, como a continuación podrá encontrarse, todos han incorporado grandes empresas en sus sistemas de comercialización con el objetivo de aumentar su presencia en los mercados:

- Rainforest Alliance estableció acuerdos comerciales con Kraft, Nestlé y McDonalds, mediante sus marcas Kenko y Maxwell House. El proyecto para Maxwell House significó comprar cinco millones de libras de café certificadas en 2004, cifra que aumentó a trece millones en 2005, las cuales se distribuyeron principalmente en Reino Unido, Francia, Suecia e Italia (Rainforest Alliance 2006b). Por otro lado, McDonalds distribuye la marca Kenko a partir de 2007 en sus 1,200 tiendas de manera exclusiva en Reino Unido e Irlanda (Rainforest Alliance 2007a). En este sistema participan además algunos de los grandes comercializadores —Ecom, Rothfos, Volcafé, Atlantic, entre otros-y grandes torrefactores como Tchibo y Gloria Jeans.

- En el sistema Common Code participan los grandes comercializadores y torrefactores mundiales de café, tales como Volcafé, Ecom Agroindustrial, Rothfos, Neumann Kaffee, Tchibo, Kraft, Nestlé, Sara Lee (Common Code 2007).

- Sara Lee, por medio de su marca Douwe Egberts, se comprometió a comprar café certificado por Utz Kapeh en 2004 debido a la presión de la organización de la sociedad civil Oxfam (Kolk 2005: 231). Además de esta empresa, participan en el sistema Rothfos, Ecom Agroindustrial, Volcafé e Illy, entre otros.

- Starbucks a partir de 2000 se comprometió a ofrecer café de comercio justo en sus tiendas, además de ofrecer un impulso a proyectos de desarrollo social en Etiopía para beneficiar a los productores; sin embargo, para 2003 el volumen de comercio justo que manejaba alcanzó poco menos de 2\% de su total comercializado (Maitland 2004). 
- También Procter \& Gamble comenzó a comprar café de comercio justo a partir de 2003 a través de su marca Millstone; posteriormente lanzó una marca de café certificado por Rainforest Alliance y en 2006 anunció tres más con certificado orgánico y de comercio justo (Millstone 2006). ${ }^{6}$

\section{LA IMPORTANCIA DE LOS SISTEMAS ALTERNATIVOS DE CAFÉ EN MÉXICO}

México es un país particularmente rico en cuanto al desarrollo de las propuestas alternativas para el café: la primera finca cafetalera con prácticas orgánicas se registró en Chiapas y el origen del sistema de sellos de comercio justo se inició en Oaxaca; ${ }^{7}$ el país además se identifica con una de las regiones de mayor biodiversidad del mundo, particularmente los espacios cafetaleros (Nestel 1995, Toledo y Moguel 1996, Moguel y Toledo 1999).

México cuenta además con una iniciativa propia, originada desde los productores, para el comercio justo, Comercio Justo México, CJM, institución encargada de la definición de una norma de comercio justo válida dentro del país, que tiene entre sus objetivos la promoción doméstica de este modelo de comercio. Surgió a finales del siglo XX por iniciativa de organizaciones importantes en la historia de sistemas alternativos cafetaleros, tales como UCIRI, ISMAM, CEPCO y Tosepan Titataniske, entre otras (CJM 2004). Sin embargo, la importancia de las iniciativas alternativas es baja en función del tamaño del sector cafetalero, confirmando así que estos sistemas son aún pequeños de nicho de mercado y solo conocidos por algunos sectores de la sociedad.

Para dimensionar esta cifra, hay que mencionar que México actualmente ocupa el séptimo lugar en cuanto a la producción mundial de café, con un volumen aproximado de 
cuatro millones y medio de sacos en $2006,{ }^{8}$ del cual 59\% se destinó a los mercados internacionales, ocupando así el lugar decimoprimero a escala mundial en exportaciones (ICO 2007b). Los destinos de las exportaciones mexicanas se distribuyeron, en 2004, en $29 \%$ para Europa y $62 \%$ para Estados Unidos, lo que representó un importante cambio respecto a las proporciones encontradas en 1989 cuando se suspendieron los Acuerdos Internacionales, que fueron de 9\% y 85\%, respectivamente (Pérez Akaki 2007: 134).

Según los datos del Censo cafetalero, en el país existen registrados casi 490,000 productores de café, en una superficie de 684,000 hectáreas, con una extensión promedio menor a 1.4 hectáreas por productor. Oficialmente se reconocen doce estados productores, aunque los registros oficiales reportan que el grano se produce en quince entidades (SIACON 2006), los cuales se muestran espacialmente en la Figura 1.

Figura 1. Municipios cafetaleros mexicanos

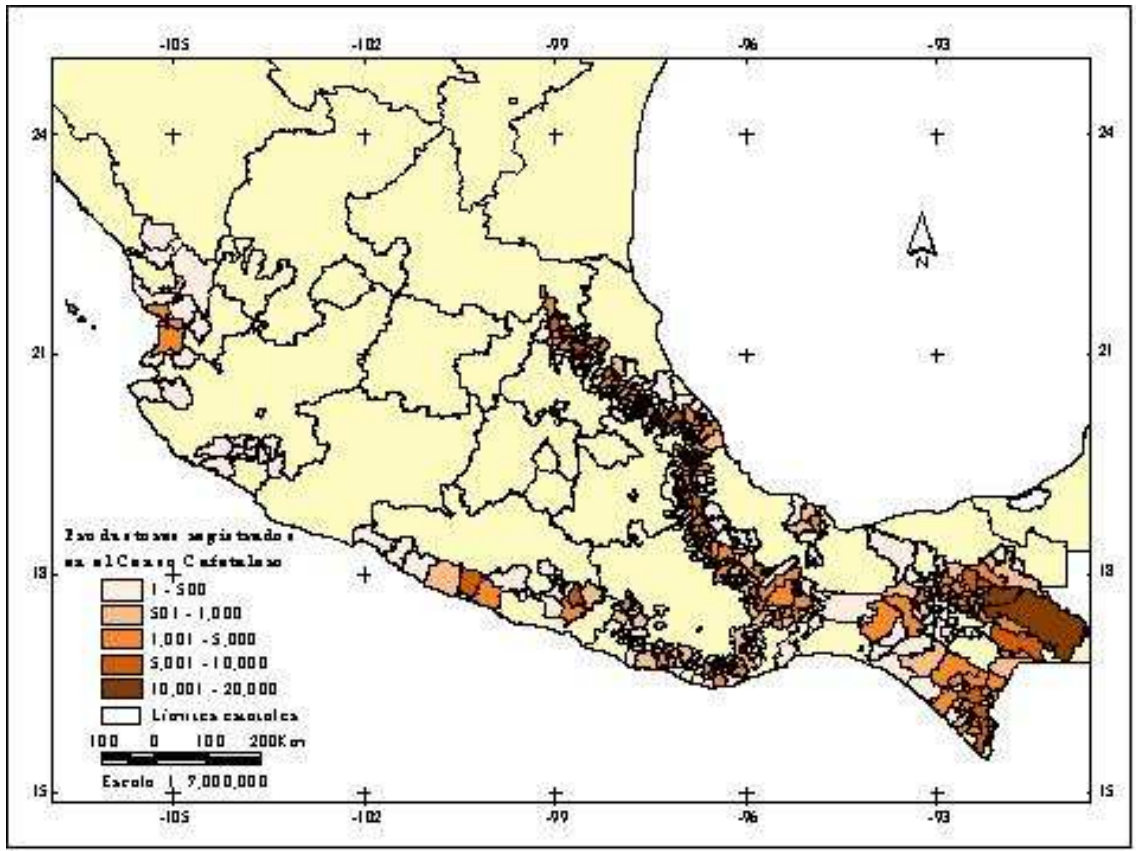

Fuente Pérez Akaki 2007: 140. 
Según la Organización Internacional del Café, ICO, México ocupó el tercer lugar mundial en producción de café orgánico en 2005, detrás de Perú y Etiopía, con una participación de mercado de $8 \%$ de la oferta mundial, equivalente a poco más de 63,500 sacos de 60 kilogramos de café verde (ICO 2006: 2).

En el Cuadro 2 se presenta un resumen de la importancia de los sistemas alternativos en México a partir de la información que ofrecen las fuentes consultadas. Vale la pena destacar que no se incluye Common Code por no existir aún estadísticas disponibles sobre sus operaciones.

Cuadro 2. Importancia de los sistemas alternativos en México

\begin{tabular}{lccc} 
Sistema & Superficie (ha) & Organizaciones & $\begin{array}{c}\text { Producción } \\
\text { (Ton) }\end{array}$ \\
\hline Orgánico & 70,045 & 184 & ND \\
Comercio Justo & $\mathrm{ND}$ & 38 & 3,744 \\
SMBC & 1,343 & 5 & 1,800 \\
Rainfores Alliance & 6,170 & 13 & 6,400 \\
Utz Kapeh & 1,813 & 2 & $\mathrm{ND}$ \\
Total & 79,371 & 242 & 11,944 \\
\hline Nota: ND - No disponible; Fuente: Elaboración propia a partir de SPC, 2007; \\
Raynolds, 2002b; FLO, 2007b; SMBC, 2007; RA, 2007b; Utz Kapeh, 2006b.
\end{tabular}

La producción de café orgánico inició en Chiapas, en fincas como Irlanda, San Miguel y Rancho Alegre, y organizaciones como ISMAM,${ }^{9}$ posteriormente se extendió hacia Oaxaca y Guerrero, a organizaciones como UCIRI y la Coalición de Ejidos de la Costa Grande, así como a otros estados del país (FIRA 2003: 39-40). En conjunto, hasta octubre de 2006 se cuantificó una superficie cafetalera destinada a la producción orgánica de 70,045 hectáreas, involucrando a 34,343 productores y 184 organizaciones (Sistema Producto Café 2007). 
En el caso del comercio justo de café, México fue el país que hasta 2006 registró el mayor número de organizaciones de productores participantes en el sistema, con 38 de un total de 231 (FLO 2007b). Según datos de FLO, hasta el año 2000 la región con mayor participación en el comercio justo fue América Latina, con 84\% del total mundial, 14,400 toneladas, y México el país con mayores volúmenes de exportación, con una proporción de $26 \%$ del total de dicho año (Raynolds 2002b).

Al hablar de los cafés de sombra o ecológicos, las cifras para México indican una superficie certificada por Rainforest Alliance de 6,170 hectáreas hasta enero de 2007, lo que equivale a una proporción de $10.5 \%$ de su superficie mundial certificada, perteneciente a 13 organizaciones (Rainforest Alliance 2007b), lo que representó una producción anual aproximada de 6,400 toneladas, equivalentes a $5.3 \%$ del total mundial producido (Rainforest Alliance 2007c). Por su parte, SMBC certificó en México hasta junio de 2007 a 226 productores, $16 \%$ del total, de 5 organizaciones, con una extensión de 1,343 hectáreas, $33 \%$ del conjunto, los cuales producen aproximadamente $40 \%$ del grano certificado total, que alcanza 1,800 toneladas anuales (SMBC 2007).

La presencia de la iniciativa Utz Kapeh en México es más modesta que las otras, pues solo se contó a marzo de 2007 con dos organizaciones certificadas, y suman una extensión de 1,813 hectáreas, proporción apenas 1.2\% de la superficie total certificada a escala mundial, aunque hay actualmente tres organizaciones más en proceso de certificación (Gamboa 2007). No existe ningún especialista en agronomía en México aprobado por Utz Kapeh para asistir a los productores que quieran obtener la certificación, 
y solo existe una institución reconocida para la certificación del producto. ${ }^{10}$ Sin embargo, las tendencias apuntan a que esta iniciativa aumentará su participación en México, dada la experiencia registrada en otros países.

\section{LAS INICIATIVAS MEXICANAS EN EL COMERCIO ALTERNATIVO DE CAFÉ}

Un caso muy relevante en la historia del comercio justo en el ámbito global fue la conformación de Comercio Justo México, CJM, institución fundada en 1999 por iniciativa de ocho organizaciones, organizaciones de productores y de la sociedad civil, entre ellas UCIRI y CNOC, ${ }^{11}$ cifra que creció para 2006 a 25 socios (Comercio Justo México 2004, Pruijn 2006). En la actualidad son miembros de Comercio Justo México organizaciones de productores bien posicionadas en el mercado de exportación como CEPCO, ISMAM, UCIRI, Majomut, Toyol Witz, CESMACH y Tosepan Titataniske, las cuales se ubican en los estados de Chiapas, Oaxaca y Puebla.

A diferencia de otras iniciativas sustentables, CJM fue creado por productores atendiendo a sus necesidades y debilidades, es decir, es una expresión local que se fundamenta en las condiciones propias de la geografía productora. Por ello fue hasta 2004 cuando recibió su afiliación a FLO Internacional, con lo cual se comprometió a buscar una definición congruente con la que este último propone a escala mundial. Sin embargo, en los primeros meses de 2007 no había una validez del sello que ofrece CJM en los mercados internacionales, por lo que los productores para exportar bajo este modelo necesitan obtener la certificación específica de FLO.

Al comparar las normas de CJM vigentes hasta abril de 2007 y las emitidas por FLO se observa un rango de mayor exigencia en las primeras, por ejemplo se observa que CJM 
establece sus criterios como obligatorios, mientras que FLO estableció algunos criterios mínimos y otros que deben mejorarse en determinado plazo de tiempo; CMJ distingue entre las diferentes calidades sensoriales de café para establecer el precio mínimo de garantía, lo cual no se definió así por FLO, sino que sólo discrimina por la especie de café y el tipo de beneficiado; ${ }^{12} \mathrm{CJM}$ además establece ciertos criterios para la calidad tanto del café tostado como del soluble basados en diferentes normas mexicanas, lo cual tampoco está definido por FLO.

Desde su aparición, CJM ha trabajado de manera cercana con Certimex, S. A. de C. V., una agencia certificadora fundada en 1997 con el propósito de que los productores agrícolas mexicanos contaran con una empresa nacional que certificara los procesos orgánicos, en ese momento a precios muy altos y solo ofrecidos por agencias internacionales. Originalmente se concentró en la certificación orgánica, posteriormente se extendió hacia la certificación de comercio justo y café de sombra. Actualmente, Certimex está acreditada por DAP, ${ }^{13}$ CAAQ, ${ }^{14}$ IFOAM y por USDA, lo cual avala que las inspecciones orgánicas son válidas tanto en Europa, como Canadá y Estados Unidos (Certimex 2007). Además cuenta con acuerdos de colaboración con Naturland, OCIA, Biolatina, IMO y Biosuisse, para los cafés orgánicos y ecológicos; FLO-CERT y CJM, para el comercio justo, y SMBC para el café amigable con las aves.

Los socios fundadores de Certimex fueron organizaciones de gran importancia como UCIRI, CEPCO y Cooperativa San Fernando, entre otras. La institución se ha interesado en mantener una importante red de contactos para colaboraciones conjuntas con la academia, el gobierno y la sociedad civil, lo que la ha llevado a trabajar conjuntamente con algunas universidades especializadas en temas rurales y con diferentes centros de 
investigación. Con el paso del tiempo, el número de organizaciones certificadas por café orgánico creció a ritmo acelerado hasta alcanzar 184 en 2006, que representan aproximadamente a 34,300 productores y superficies ligeramente superiores a 70,000 hectáreas (Sistema Producto Café 2007). En el año 2000, el comercio justo representó 37,000 hectáreas y 18,000 productores. Desde ese año su crecimiento anual promedio alcanzó $11.2 \%$ en superficies y 10.6\% en productores (Sistema Producto Café 2007).

La ubicación geográfica de los espacios productivos cafetaleros orgánicos en México se encuentra principalmente en Chiapas y Oaxaca, aunque también se produce grano orgánico en Tabasco, Veracruz, Puebla, Guerrero y Jalisco. ${ }^{15}$ No hay iniciativas certificadas por Certimex en el resto de estados donde oficialmente se produce café (Martínez 2007).

Junto con Certimex, CJM impulsó la creación de la empresa Agromercados en 2000 (CJM 2004: 24), a la cual se le encargó la elaboración y comercialización de productos bajo la figura de comercio justo, certificados por Certimex bajo la norma de CJM. Esta empresa desarrolló la marca de café Fértil, producto pionero utilizado para la promoción del sistema de comercio justo en el país. En Agromercados participan las mismas organizaciones mencionadas, por ello Fértil es una mezcla de granos orgánicos de Chiapas, Oaxaca y Puebla, que se ofrecen en presentación de café tostado y soluble. Las ventas de café Fértil en México se aprecian en la Figura 2, que muestran una tasa de crecimiento anual promedio de $75 \%$.

En la actualidad, el sistema de comercio justo impulsado en México tiene una red de distribución de sus productos de aproximadamente 50 puntos, la gran mayoría son cafeterías pequeñas y ubicadas en la zona metropolitana de la ciudad de México, Chiapas y 
Morelos, aunque se firmó recientemente un convenio para que el café Fértil se pueda comercializar en dos tiendas de autoservicio, lo cual podrá hacer más grande la distribución del producto y llevarlo por ende a más consumidores. ${ }^{16}$

A pesar de estos esfuerzos, el número de organizaciones certificadas por CJM no ha crecido en los últimos años. Una de las razones es la necesidad del reconocimiento de FLOCert cuando se desea exportar a Europa, pues el certificado de CJM no es válido ni la marca reconocida en los mercados internacionales. Otra es la poca importancia que tiene el sistema de comercio justo en el territorio nacional, lo cual puede confirmarse por los volúmenes tan bajos que maneja y la limitada capacidad de distribución, aspecto que está en parte relacionado con los bajos volúmenes de consumo de café en el país (ASERCA 2002: 37). Si a este elemento se suma que en algunos aspectos la certificación de CJM es más estricta que la de FLO, se tiene un motivo más para explicar este resultado.

Figura 2. Ventas de café Fértil en México (kilogramos)

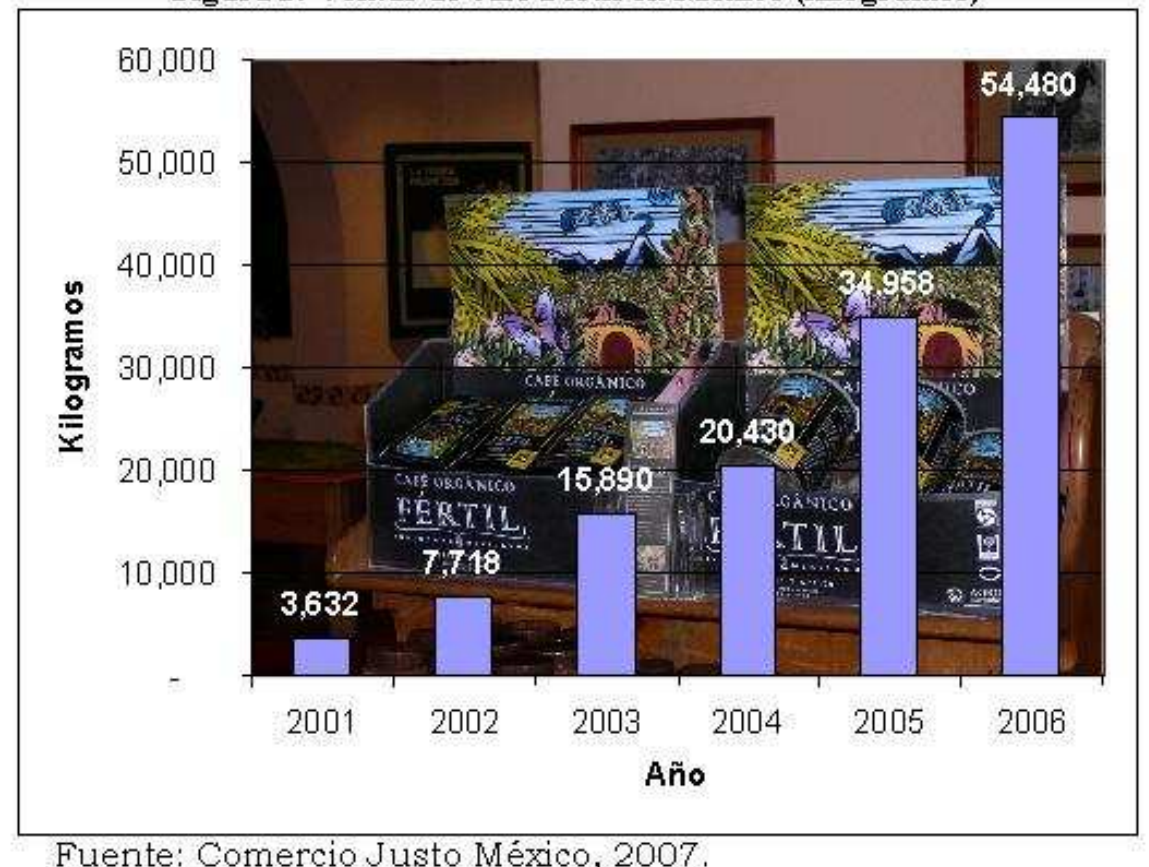

Fuente: Comercio Justo México, 2007. 


\section{LA TOSEPAN TITATANISKE Y SU INCORPORACIÓN AL COMERCIO JUSTO}

El origen de la cooperativa Tosepan Titataniske se registró en 1977 a partir de las dificultades que los habitantes de la región de Cuetzalan, Puebla, encontraban para poder mejorar su calidad de vida; desde su fundación se eligió un enfoque sustentable de producción agropecuaria, forestal y de servicios. Con el paso de los años, la organización de productores ha tenido objetivos específicos acordes con las preocupaciones más apremiantes de los miembros: durante los ochenta fue gestora de recursos federales para los socios, después fue la responsable de la aplicación de los recursos federales para el desarrollo regional y posteriormente se convirtió en una organización apartada de la política orientada exclusivamente a las actividades productivas (Bartra, Cobo y Paredes 2004: 3748).

Desde la década de los setenta el café es el principal producto comercial de la región, impulsado por el Instituto Mexicano del Café. Inmecafé, y así sustituyó paulatinamente a la caña de azúcar. Sin embargo, tras los cambios en la regulación internacional en 1989 que llevaron a un desplome en los precios y aunado a una helada que se registró en la región en esas fechas, se impulsó un proceso de diversificación productiva que significó, por un lado, la adopción de prácticas sustentables de producción agropecuaria, entre ellas la producción de pimienta, mamey, macadamia, cedro rojo, caoba, bambú y miel, aunque según reconoce su asesor, «el café sigue siendo el principal producto de la cooperativa» ${ }^{17}$; y por el otro, proyectos sustentables de ecoturismo, como un hotel y un centro de formación con sistemas innovadores de reciclaje de agua; proyectos de 
mujeres orientados a las artesanías y la producción de alimentos; una caja de ahorro y crédito; proyectos de mejoramiento de viviendas; de comercialización de café y otros productos, y un centro de formación para organizaciones de productores agrícolas (Tosepan Titataniske 2007).

La cooperativa cuenta en la actualidad con 1,304 socios activos, entre ellos $75 \%$ son productores de café, quienes cultivan 1,290 hectáreas dedicadas a la producción del grano, de las cuales $46 \%$ están certificadas como orgánicas. Esto representa una superficie de 0.99 hectáreas por socio y un promedio de 1.7 parcelas por socio, lo cual significa que los terrenos además de ser pequeños están fraccionados (Duran 2007). Cuando se contabilizan además los socios no activos, incluyendo los miembros de la caja de ahorro, se habla de más de 4,000 familias involucradas en 6 municipios que mayoritariamente son indígenas náhuatl (Durán 2006).

A partir del año 2000 la cooperativa inició el proceso de certificación de sus productos agrícolas, café y pimienta, con el objetivo de participar en el sistema orgánico y de comercio justo. De esta forma, en 2002 obtuvo la certificación de OCIA, en 2003 de Certimex, tanto por orgánicos como por comercio justo, y en 2006 se iniciaron los trámites para la certificación ante FLO (Durán 2007).

Desde los ochenta se creó la marca de café tostado y molido Tosepan, que desde 2003 se vende bajo el modelo de comercio justo pero con una red muy limitada y sólo en la región donde se produce el grano. A partir de 2007 este producto se ofrece en la ciudad de México en un par de tiendas orgánicas y, aunque la mayor parte de su producto se exporta a mercados como Estados Unidos y Japón, como un café de especialidad, se pretende concretar la exportación por primera vez a Europa bajo el sistema de comercio justo. 
Los miembros de la cooperativa reconocen que gracias a la diversificación productiva han podido sostenerse por casi treinta años, evitando la dependencia exclusiva en el café y desarrollando diferentes proyectos sustentables, que además de garantizar el cuidado del ambiente ofrecen estabilidad en los ingresos y promueven la integración de los productos y servicios ofrecidos. Reconocen asimismo que la seguridad de precios que les brinda la participación en el comercio justo es un elemento fundamental para dar certidumbre a sus ingresos y así poder tomar mayores riesgos al iniciar otros proyectos.

\section{CONCLUSIONES}

La aparición de los sistemas alternativos para la producción y comercialización de café se dio a partir de varios factores coyunturales: la inviabilidad del sistema de cuotas a las exportaciones mundiales de café, con la consecuente crisis que se generó tras la liberalización de mercado, la entrada de nuevos participantes, los cambios en las fuerzas políticas alrededor del grano y la segmentación de los gustos de los consumidores de café en todo el mundo, que además afectó al sector alimentario en conjunto.

Ante esto, los sistemas alternativos más antiguos, orgánico y comercio justo, comenzaron su rápido crecimiento durante los ochenta, pero con dos objetivos muy diferentes: el primero buscando atender la demanda creciente en los países desarrollados por la sanidad y la calidad en los alimentos; el segundo, intentando un modelo incluyente con mayor equidad para los productores que permitiera la mejoría en sus condiciones de vida. Los sistemas sustentables que aparecieron después han tomado un camino similar al de los orgánicos, orientándose a criterios técnicos en producción agrícola, pero manteniendo a un nivel mínimo los criterios sociales, aunque una característica muy 
importante de todos ellos es el uso — en algunos casos abuso- de la mercadotecnia para la promoción de un «consumo responsable» que incorpore algunos valores éticos (Doppler y González 2007).

Paradójicamente, los criterios sociales parecen resultar inalcanzables por los actores dominantes en las cadenas de comercialización, ya sean los comercializadores o los torrefactores, al grado que reconocen que muchos de estos criterios deben ser relajados, lo cual es acorde con la actual presión social para eliminar restricciones en los mercados laborales y hacerlos más flexibles. Esta lógica lejos de beneficiar a productores y trabajadores puede acentuar aún más las diferencias en los ingresos y generar una mayor inequidad social (Harvey 2006). De entre los sistemas comparados, el de comercio justo es el que mayor énfasis pone en aspectos sociales. Los restantes sistemas se orientan a otras dimensiones y la social no es tan relevante, pues se confía en el mercado para otorgar la equidad prometida.

Entre las diversas críticas a los sistemas alternativos, la participación de grandes trasnacionales aparece como una constante, y como una amenaza eventual para alcanzar sus objetivos de sustentabilidad. Aunque los sistemas SMCB, Rainforest Alliance, 4C y Utz Kapeh, declaran la búsqueda de sustentabilidad para los productores cafetaleros, su alcance queda cuestionado, algunos por el mercado que atienden, y porque en sus reglas no se define de manera clara un pago más equitativo para los actores de la cadena, lo que podría convertirse entonces en nuevos arreglos, o nuevas convenciones, en la cadena tradicional del café. Sin embargo, estas convenciones se crearon en países consumidores no en los productores, con lo cual el rango de participación que tienen los productores es mínimo. 
Es por ello que la sociedad civil resulta de gran importancia para buscar acuerdos que traten de ofrecer mayor equidad en los participantes. En este punto el modelo de comercio justo encuentra otra bondad: ser más incluyente en cuanto a las iniciativas de todos los actores involucrados en el comercio, aunque se pueden hacer aún muchas mejoras en este sentido. Otras críticas sobre este sistema son la asimetría de fuerzas entre los participantes, la imposición de normas desde los países desarrollados y la exclusión como una condición, dado su funcionamiento bajo las reglas del mercado (González, Linck y Moguel 2003, Doppler y González 2007).

La Tosepan Titataniske hace evidentes las ventajas que comercialmente puede ofrecer el comercio justo, pero también deja ver la importancia de la diversificación productiva. El precio garantizado funciona como un seguro para los ingresos, y la posibilidad de compra anticipada de la producción es un mecanismo de capitalización que por otros medios no sería posible. Sin embargo, la experiencia de esta organización demuestra que el comercio justo solo es una parte de la estrategia y resulta insuficiente para atenuar los problemas económicos de los productores.

Ante esta evidencia, los sistemas alternativos se presentan como propuestas importantes, aunque aún insuficientes para mejorar la vida de los productores de café en el mundo. 


\section{BIBLIOGRAFÍA}

Apoyos y Servicios a la Comercialización Agropecuaria, Aserca, 2002, «Café de México». Claridades Agropecuarias, núm. 103. SAGARPA, México.

Bartra, Armando, Rosario Cobo y Lorena Paz Paredes, 2004, Tosepan Titataniske. Abriendo horizontes, 27 años de historia. Sociedad Cooperativa Agropecuaria Regional Tosepan Titataniske, S. C. L., México.

Biggart, Nicole W. y Thomas D. Beamish, 2003, «The economic sociology of conventions: Habit, custom, practice and routine in market order». Annual Review of Sociology, vol. 29 , pp. 443-464.

Boyer, Robert, 2002, «From canonical Fordism to different modes of development». En Regulation Theory. The State of the art, editado por Robert Boyer y Yves Saillard, pp. 231-237. Routledge, Nueva York.

Busch, Lawrence y Bain, Carmen, 2004, «New! Improved? The transformation of the global agrifood system». Rural Sociology, vol. 69, núm. 3, pp. 321-346.

Cámara de Diputados, 2006, «Café, la agroexportación». Documento base para el Foro Café, la agroexportación, 7 y 8 de junio.

Certificadora Mexicana-Certimex, 2007, «Acreditaciones». En http://www.certimexsc.com/ quienes_acreditaciones.htm [consulta: marzo, 2007].

Choi, Young B., 1999, «Conventions and economic change: A contribution toward a Theory of Political Economy». Constitutional Political Economy, vol. 10, pp. 245264. 
Comercio Justo México, CJM, 2004, Comercio Justo. El poder de un mercado diferente. Informe de actividades 1999-2004. México.

Common Code for the Coffee Community, 2004, Código Común para la Comunidad Cafetalera, versión 9 de septiembre de 2004.

2007, Members of the Common Code for the Coffee Community Association, febrero.

Daviron, Benoit y Stefano Ponte, 2005, The Coffee Paradox. Global markets, commodity trade and the elusive promise of development. Zed Books, Londres, Reino Unido.

Doppler, Flurina y Alma Amalia González, 2007, «El Comercio Justo: Entre la institucionalización y la confianza». Problemas del Desarrollo, vol. 38, núm. 149. UNAM, México.

Durán, Leonardo, 2006, «Sociedad Cooperativa Agropecuaria Regional Tosepan Titataniske». Ponencia presentada en el Foro Café: la agroexportación, 7 y 8 de junio, mecanuscrito.

2007, Asesor de la Cooperativa Tosepan Titataniske. Comunicación personal, 17 de febrero.

Escamilla Prado, Esteban y Octavio Ruiz Rosado, 2006, «Producción y calidad del café orgánico en México». Ponencia presentada en el Foro Café: la agroexportación, 7 y 8 de junio, mecanuscrito

Fairtrade Labelling Organizations, FLO, International, 2007a, Criterios genéricos de comercio justo fairtrade para organizaciones de pequeños productores. FLO internacional. 
2007b, Number of fairtrade coffee producers per country. En http://www.fairtrade.net/ coffee.html [consulta: marzo, 2007].

Fideicomisos Instituidos en Relación a la Agricultura, FIRA, 2003, «Agricultura orgánica, Una oportunidad sustentable de negocios para el sector agroalimentario mexicano». FIRA Boletín informativo, vol. XXXV, núm. 322, diciembre.

Fridell, Gavin, 2006, «Comercio justo, neoliberalismo y desarrollo rural: una evaluación histórica». Íconos, núm. 24, pp. 43-57.

Gigante, 2007, «Perfil Corporativo». En http://www.gigante.com.mx [consulta: febrero, 2007].

Giovannucci, Daniele y Freek Jan Koekoek, 2003, The State of Sustainable Coffee: A study of twelve major markets. International Coffee Organization e International Institute of Sustainable Development, Londres, Inglaterra.

Giovannucci, Daniele y Stefano Ponte, 2005, «Standards as a new form of social construct? Sustainability initiatives in the coffee industry». Food Policy, vol. 30, pp. 284-301.

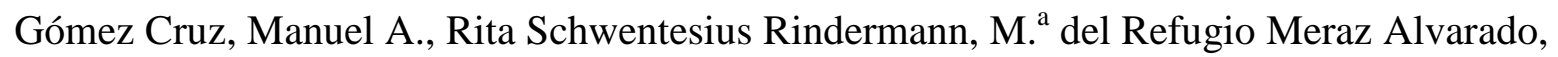
Aurora J. Lobato García y Laura Gómez Tovar, 2005, Agricultura, apicultura y ganadería orgánicas de México-2005. Situación-retos-tendencias. PIAICIESTAAM, México.

González, Alma Amalia, Thierry Linck y Reina Moguel, 2003, «El comercio de valores éticos: Las reglas del juego del café solidario». Revista Europea de Estudios Latinoamericanos y del Caribe, núm. 75, pp. 31-45. 
González, Alma Amalia y Ronald Nigh, 2005, «Smallholder participation and certification of organic farm products in Mexico». Journal of Rural Studies, vol. 21, pp. 449460.

Greenberg, Russell, 2001, «Overview of sustainable coffee». En http://nationalzoo.si.edu/ ConservationAndScience/MigratoryBirds/Coffee/thoughtpaper.cfm [consulta: marzo, 2007].

Harvey, David, 2006, «Neo-liberalism as creative destruction». Geografiska Annaler, Series B: Human Geography, vol. 88, núm. 2, pp. 145-158.

International Coffee Organization, ICO, 1997, Agricultural and Economic Analysis of Organically Grown or «Organic» Coffee. Londres, Inglaterra.

2006, Organic Coffee Export Statistics. Calendar Year 2005. Londres, Inglaterra.

2007a, «Total production of exporting countries. Crop years 2001/02 to 2006/07». En http://www.ico.org [consulta: marzo, 2007].

2007b, «Exports by exporting countries to all destinations». En http://www.ico.org [consulta: marzo, 2007].

International Federation of Organic Agriculture Movements, IFOAM, 2005, «Principles of Organic Agriculture». En http://www.ifoam.org [consulta: diciembre, 2006].

Kilcher, Lukas, 2005, «How organic agriculture contributes to sustainable development». En The World of Organic Agriculture. Statistics and Emerging Trends 2005, editado por Helga Willer y Minou Yuseefi. IFOAM, Alemania. 
Kolk, Ans, 2005, «Corporate Social Responsability in the coffee sector: The dynamics on MNC responses and Code Development». European Management Journal, vol. 23, núm. 2, pp. 228-236.

Krier, Jean-Marie, 2005, Fair Trade in Europe 2005. Facts and figures on Fair Trade in 25 countries. FLO, IFAT, NEWS! y EFTA, Bélgica.

Lewis, David, 1969, Convention: A philosophical study. Harvard University Press, Massachussets, Estados Unidos.

Maitland, Alison, 2004, «Starbucks tastes Oxfam’s brew». Financial Times, 14 de octubre de 2004, pp. 12.

Millstone, 2006, «Millstone ${ }^{\circledR}$ Coffee expands Commitment to Social Responsibility with three new organic, Fair Trade Certified ${ }^{\mathrm{TM}}$ Blends». En http://www.millstone.com [consulta: marzo, 2007].

Moguel, Patricia y Víctor Toledo, 1999, «Biodiversity conservation in traditional coffee systems of Mexico». Conservation Biology, núm. 13, pp. 11-21.

Morgan, Kevin y Jonathan Murdoch, 2000, «Organic vs. conventional agricultural: knowledge, power and innovation in the food chain». Geoforum, vol. 31, pp. 159173.

Muradian, Roldan y Wim Pelupessy, 2005, «Governing the Coffee Chain: The Role of Voluntary Regulatory Systems». World Development, vol. 33, núm. 12, pp. 20292044. 
Murdoch, Jonathan, Terry Marsden y Jo Banks, 2000, «Quality, nature and embeddedness: some theoretical considerations in the context of the food sector». Agricultural and Human Values, vol. 17, núm. 1, pp. 107-125.

Naturland, 2005, Normas de Naturland, Producción. Alemania.

Nestel, David, 1995, «Coffee in Mexico: international market, agricultural landscape and ecology». Ecological Economics, núm. 15, pp. 165-178.

Oechssler, Jörg, 1999, «Competition among conventions». Computational \& Mathematical Organizational Theory, vol. 5, núm. 1, pp. 31-34.

Pendergrast, Mark, 2002, El café. Historia de la semilla que cambió el mundo. Javier Vergara Editor, Argentina.

Perfecto, Ivette, Robert Rice, Russell Greenberg y Martha E. van der Voort, 1996, «Shade Coffee: A Disappearing refuge for Biodiversity». Bioscience, vol. 46, núm. 8, pp. 598-608.

Pérez Akaki, Pablo, 2007, «Los pequeños productores de café de la región Otomí-Tepehua: su problemática y sus alternativas». Tesis doctoral. UNAM, México.

Ponte, Stefano, 2002, «Standards, Trade and Equity: Lessons from the Speciality Coffee Industry». CDR Working Paper, núm. 02.13. Copenhague.

Pruijn, Jeroen, 2006, «Café de comercio justo, el poder de un mercado diferente». Ponencia presentada en el Foro Café: la agroexportación, 7 y 8 de junio.

Rainforest Alliance, 2005, Sustainable Agriculture Standard. Rainforest Alliance, EUA. 
2006a, About us. Our Mission. En http:// www.rainforest-alliance.com [consulta: diciembre, 2006].

2006b, Profiles in Sustainability: Kraft Takes the Lead in Supporting Sustainable Coffee Production. Nueva Cork, EUA.

2007a, Reflecting Trend Toward Global Companies Embracing Sustainability, McDonald's UK Puts Rainforest Alliance Certified Coffee on the Menu. En http://www.rainforest-alliance.org/news.cfm?id=mcdonalds [consulta: marzo, 2007].

2007b, Summary of certified area under cultivation. En http://www.rainforestalliance.org/programs/agriculture/shop/documents/certified_farms.pdf [consulta: marzo, 2007].

2007c, Products from Farms Certified by the Rainforest Alliance-Coffee Producers. En http://www.rainforest-alliance.org/programs/agriculture/shop/coffeeproducers_mexico.html [consulta: marzo, 2007].

Raynolds, Laura, 2002a, «Consumer/Producer Links in Fair Trade Networks». Sociologia Ruralis, vol. 42, núm. 4, pp. 404-424.

2002b, «Poverty alleviation through participation in fair trade coffee networks: existing research and critical issues». Documento para la Fundación Ford, disponible en www.colstate.edu/depts/sociology/FairTradeResearchGroup/doc/rayback.pdf. (Fecha de consulta: 7 de febrero de 2007).

Reardon, Thomas y Christopher Barret, 2000, «Agroindustrialization, globalization, and international development. An overview of issues, patterns and determinants». Agricultural Economics, núm. 23, pp. 195-205. 
Renard, Marie-Christhine, 1999, Los intersticios de la globalización. Un label (Max Havelaar) para los pequeños productores de café. Misceláneas, México, D. F.

Rice, Robert A. y Justin R. Ward, 1996, Coffee, Conservation, and Commerce in the Western Hemisphere. Smithsonian National Zoological Park, Estados Unidos.

Rindova, Violina P. y Charles J. Fombrun, 2001, «Entrepreneurial action in the creation of the specialty coffee niche». En Entrepreneurial Dynamic: The origins of entrepreneurial and its role in industry evolution, Claudia Schoonhoven y Elaine Romanelli (eds.), pp. 236-261. Estados Unidos.

SETEM, 1999, Café amargo. Por un comercio Norte-Sur más justo. Icaria-Más Madera, Barcelona, España.

Sistema de Información Agroalimentaria de Consulta, SIACON, 2006, versión 1.1. Secretaría de Agricultura, Ganadería, Desarrollo Rural y Pesca, México.

Sistema Producto Café, 2007, Correo Cafetalero. núm. 576, 26 de abril.

Smithsonian Migratory Bird Centre, SMBC, 2002, Norms for Production, Processing and Marketing of Bird Friendly Coffee, Versión 1.

2007, Bird friendly coffee. En http://nationalzoo.si.edu/ConservationAndScience/ MigratoryBirds/Coffee/Farms [consulta: enero, 2007].

Superama, 2007, «Directorio de tiendas». En http://www.superama.com.mx [consulta: febrero, 2007]. 
Tosepan Titataniske, 2007, «¿A qué nos dedicamos?». En http://www.tosepan.com [consulta: febrero, 2007].

Toledo, Víctor y Patricia Moguel, 1996, «En busca de un café sostenible en México. La importancia de la diversidad biológica y cultural». Ponencia presentada al Primer congreso del café sostenible Smithsonian Migratory Bird Centre, Washington, Estados Unidos, 16-18 de septiembre, mecanuscrito.

UTZ Kapeh, 2006a, Utz. Kapeh Código de conducta. Versión 2006 rev.01.

2006b, Utz Kapeh Annual Report 2005. Utz Kapeh, Ámsterdam, Holanda.

2007a, «List of independet certifiers». En http://www.utzcertified.org /index.php [consulta: marzo, 2007].

2007b, «Trained agronomist list». En http://www.utzcertified.org/index.php [consulta: marzo, 2007].

Watts, Michael y David Goodman, 1997, «Agrarian questions, global appetite, local metabolism: nature, culture, and instrustry in fin-de-cycle agrofood systems». En Globalization, food, agrarian questions and global restructuring, editado por D. Goodman y M. Watts, pp. 1-34. Routledge, Estados Unidos.

Young, H. Peyton, 1993, «The evolution of conventions». Econometrica, vol. 61, núm. 1, pp. 57-84. 


\section{Notas}

${ }^{1}$ Algunos consideran que en este grupo también se debe incluir el café de especialidad, pero no se ha inscrito en esta lista por centrarse de manera exclusiva en la calidad, sin presentar alguna otra innovación como las de los otros sistemas productivos.

${ }^{2}$ Aunque esta última solo es aplicable a algunos productos, no para el caso de perennes como el café.

${ }^{3}$ Naturland fue creada en 1982 y OCIA en 1985.

${ }^{4}$ Raynolds (2002b) incluso reporta tasas cercanas a $30 \%$ en los primeros años.

5 Sustainable Agriculture Network.

${ }^{6}$ Aunque en la página oficial de las marcas de café solo especifica que se trata de un café orgánico, no de comercio justo.

${ }^{7}$ La Finca Irlanda en Chiapas goza del reconocimiento mundial que sus prácticas orgánicas comenzaron desde la década de los cincuenta. Por otro lado, la iniciativa Max Havelaar surgió en 1988 vinculando a la cooperativa UCIRI, Unión de Comunidades Indígenas Rurales del Istmo, en Oaxaca con los mercados europeos (SETEM 1999: 78, Renard 1999: 182-183, FLO 2007b).

${ }^{8}$ Sacos de $60 \mathrm{~kg}$ de café verde (ICO 2007a).

${ }^{9}$ Indígenas de la Sierra Madre de Motozintla, con sede en Tapachula, Chiapas.

${ }^{10}$ Tan solo en Guatemala existen 29 agrónomos entrenados y certificados para apoyar a los productores que quieran adquirir la certificación (Utz Kapeh 2007a, 2007b).

${ }^{11}$ La Coordinadora Nacional de Organizaciones Cafetaleras es uno de los organismos independientes más importantes que organiza a los productores cafetaleros.

12 En CJM se establecieron seis calidades del café, de acuerdo con la norma mexicana NMX-F-551-1996. Para FLO se discriminan entre cafés arábigas y robustas, y en cada caso entre lavados y no lavados, además de las regiones de donde proviene el café.

${ }^{13}$ Deutsches Akkreditierungssystem Prüfwesen GMBH, un organismo alemán de acreditación, con validez en la comunidad europea.

${ }^{14}$ Conseils des Apellations Agroalimentareis du Québec, en Canadá.

${ }^{15}$ No se pudieron obtener las proporciones actualizadas de cada estado en la producción nacional, pero de acuerdo con Certimex, en 2005 Chiapas poseía $58 \%$ de la superficie orgánica nacional, Oaxaca $31 \%$, Guerrero 6\%, Veracruz 4\% y Puebla 2\%, lo cual involucró a 145 municipios y 1,251 comunidades (citado en Escamilla y Ruiz 2006).

${ }^{16}$ Se trata de las empresas Gigante y Superama. La primera es de capital mexicano, cuenta con 22 tiendas en la zona metropolitana de la ciudad de México y tiene el formato de un hipermercado. El convenio es para distribuirla en diez tiendas. La segunda es una tienda de conveniencia del grupo Walmart con trece sucursales en la ciudad de México. Esta se distingue por la variedad de su oferta y por su orientación hacia sectores económicos de mayores ingresos (Gigante 2007, Superama 2007).

${ }^{17}$ Particularmente en el caso de la pimienta gorda, la Tosepan tiene una experiencia única al desarrollar el concepto de pimienta orgánica y así posicionar a México en un ámbito mundial de calidad en torno a este producto.

Fecha de recepción: 05 de febrero de 2008.

Fecha de aceptación: 01 de julio de 2008. 